\title{
TRAS LAS HUELLAS DE ALTERNATIVAS DIDÁCTICAS PARA LA ENSEÑANZA DE LA FILOSOFÍA
}

\author{
Esperanza Camargo Camargo \\ Leidy Jazmín Barreto Bernal \\ Escuela de filosofía \\ UPTC
}

\section{Introducción}

$\mathrm{E}$

n este texto presentamos algunos planteamientos acerca de la enseñanza de la

filosofía en la educación media, derivados de dos prácticas de campo en las que se proponía diseñar, aplicar, evaluar y contrastar dos propuestas metodológicas bajo un enfoque constructivista implementadas en dos instituciones educativas, a partir de los desarrollos teóricos de Miguel Ángel Gómez Mendoza y de José Joaquín García García. Partimos de tener en cuenta que enseñar filosofía no es solamente enseñar conceptos, sino que es también enseñar un modo de vida, de pensar, de dudar y de promover un cierto inconformismo con el pensamiento común, aspirando a un desarrollo autónomo y cognitivo del estudiante; es por ello que consideramos viable promover estas estrategias puesto que buscan un progreso en la calidad de la enseñanza de la filosofía, promoviendo así una nueva visión de lo que ésta ha sido y de lo que es en la actualidad.

La educación del pensar debe reposar necesariamente sobre una práctica reflexiva y crítica que logre un ejercicio permanente de la razón en el joven, de tal manera que aprenda a replantear los problemas de su entorno y de la misma manera tener la capacidad de solucionarlos. La filosofía es el ejercicio que puede desarrollar en el estudiante un pensamiento que se conecte con su entorno o con su realidad, y el encargado de construir y promover estas habilidades es el maestro. Así, "la pregunta por una estrategia didáctica es el surgimiento del pensamiento del otro, por eso enseñar/aprender filosofía (a filosofar) es una tarea compartida" ${ }^{1}$. El maestro siempre debe tener en cuenta a los jóvenes que están sentados al frente de él, sujetos que se están formando y que necesitan una buena guía: qué mejor conductor que un maestro que tiene como manual los conocimientos filosóficos y que puede mostrar el camino al estudiante para que piense por sí mismo.

Toda filosofía es de alguien y para alguien; aunque la filosofía respeta la individualidad del estudiante debe partir de problemas actuales para que el estudiante desarrolle sus capacidades de reflexión y aprenda a argumentar acerca de los conflictos de su propia realidad. En filosofía se deben diseñar estrategias pedagógicas-metodológicas que consigan

1 CERLETTI, Alejandro. Enseñar filosofía: de la pregunta filosófica a la propuesta metodológica. En: Revista Novedades Educativas. Argentina No. 169. (Enero. 2005); P.14 
promover dichas competencias en el estudiante, por lo tanto nuestro propósito es aplicar métodos didácticos que faciliten el desarrollo de procesos cognoscitivos del educando.

El objetivo principal de nuestro trabajo monográfico y de este informe radica en el estudio de dos estrategias didácticas, la Disertación filosófica y la Enseñanza por resolución de problemas, modelos pedagógicos sobre los cuales se hicieron prácticas en dos instituciones diferentes: el Colegio de Boyacá de Tunja y el Instituto Técnico Rafael Uribe de Toca (Boyacá) pues la una es estrictamente académica mientras que la otra se dirige por un enfoque técnico agropecuario, evidenciándose las diferencias de ámbitos sociales y culturales, por lo tanto la implementación de los métodos tuvo que adecuarse a las necesidades de dichos planteles educativos. A continuación presentamos un pequeño bosquejo sobre la dos metodologías aplicadas.

\section{DISERTACIÓN FILOSÓFICA}

La disertación filosófica pretende despertar el interés del estudiante para que asuma actitudes frente a problemas que lo toquen de cerca o que puedan ser interesantes para la sociedad en la que se desenvuelve. Solo cuando un estudiante encuentra la relación entre los problemas conceptuales y los de su propia experiencia, podrá desarrollar perspectivas filosóficas que lo conduzcan al progreso de su pensamiento.

Disertar: Se define como razonar detenida y metódicamente sobre alguna materia. Según Gómez Mendoza hay una diferencia radical entre disertar y disertación, en cuanto a que disertar se puede entender como la dedicación a un tema cualquiera en donde es necesario investigar a fondo una serie de autores para argumentarlo. En la disertación no es necesario conocer todo lo dicho sobre tema alguno, sólo se necesita leer detenida y cuidadosamente un tema de interés donde podamos de manera seria aportar nuestros argumentos, aunque hay que aclarar que no consiste simplemente en opinar o en especular vanamente, por el contrario en una disertación de calidad la persona puede poner a prueba su capacidad de comprensión, análisis, significación, entre otras. Otra diferencia radica en que la disertación conlleva necesariamente incluido un método; entendiendo como método aquel procedimiento por el cual pretendemos alcanzar un fin, para el que se establecen ciertos pasos o mecanismos que permitan lograr el objetivo propuesto. Gómez Mendoza nos propone otros pasos, anteriores a la disertación filosófica, igual de importantes. Para llegar a una buena comprensión del tema se deben recoger por escrito brevemente los siguientes apartados:

- Materias que entran o se relacionan con el tema

- Inventario de los términos más importantes que aparecen y otros relacionados con ellos

- Análisis de los términos más importantes que se han señalado.

- Investigar las soluciones o planteamientos históricos que se conocen sobre el tema. 


\section{Método}

\section{- Formulación del tema o introducción:}

"El punto de partida de una disertación filosófica es un tema, generalmente formulado bajo la forma de una pregunta" ${ }^{2}$. Existe una cierta clase de preguntas cuya respuesta remite a nuestra realidad, es decir, con sólo observar podemos llegar a una solución, y se conocen como preguntas factuales. En cambio hay otras que expresan un problema concretamente, son aquellas que no obtienen respuesta en la mera observación de los hechos, ya que pueden conllevar una multiplicidad de respuestas contrarias. En filosofía generalmente nos enfrentamos al segundo tipo y por lo tanto serán las preguntas más frecuentes en los temas de disertación filosófica. Al respecto conviene decir que la función del maestro radica en poder concentrar en una sola pregunta un problema fundamentalmente filosófico, que despierte las capacidades de raciocinio de sus alumnos.

Gómez Mendoza define el problema como una contradicción, es decir dos proposiciones que parecen verdaderas, que se pueden argumentar separadamente, pero que pueden oponerse la una a la otra, de tal suerte que si la una es verdadera la otra es falsa. Por lo tanto es importante concluir que es imposible sustentar simultáneamente las dos ideas incompatibles; es así que pondremos un continuo ejercicio de pensamiento a nuestro alumno puesto que debe seleccionar y argumentar lógicamente algunas de las dos salidas al problema. Para tratar de resolverla se deben llevar a cabo dos operaciones: primero, hallar las reformulaciones posibles de la pregunta con el fin de encontrar el sentido y luego, definir los términos de la pregunta "todos sin excepción".

Los pasos a seguir son: reformular la pregunta planteada a partir de la definiciones; introducir la pregunta en situación, es decir, buscar en los hechos, en nuestra realidad, ejemplos que nos ayuden a aclarar en qué situaciones se ha presentado este problema; preguntarnos cuáles son las opiniones comunes que se tienen sobre el tema, si estas opiniones no concuerdan esto sugiere una contradicción. Por último empezar a construir hipótesis que nos conduzcan a una posible respuesta, preguntarnos constantemente cuál es su implicación, por qué se supone que esta es la más acertada, cuáles son sus consecuencias. Ahora, si aparece una contradicción en las respuestas, es necesario exponerlo en el escrito y demostrar cómo se llegó al conflicto u oposición o contradicción.

- Desarrollo:

Se trata de plantear lo que es el cuerpo del trabajo, siempre siguiendo un plan, que no es un artificio de presentación sino una necesidad para desarrollar nuestro pensamiento; tampoco existen planes mágicos para todos los problemas puesto que cada problema exige un tratamiento particular. Entonces, se debe distinguir claramente cuál es la problemática, luego se elaboran varias soluciones sucesivas (siempre teniendo en cuenta que estas parten de la problemática), pues el objetivo nunca será encadenar una serie de problemas, todas las posibles soluciones deben ser argumentadas y disferenciadas y, por

2 GOMÉZ MENDOZA, Miguel Ángel. Introducción ala didáctica de la filosofía. Papiro. Pereira, Colombia. 2003. P.47. 
último, se debe ser siempre progresivo y riguroso, saber ordenar nuestro trabajo de manera en que se observe fácilmente nuestro proceso de pensamiento.

En el cuerpo del trabajo no son de gran importancia las opiniones sino las razones por las cuales llegamos a una solución, es necesario dar ejemplos pero estos deben ser concretos y analizados respectivamente, pues es claro que un ejemplo en estado bruto no prueba nada. Recurrir a algún filosofo o figura importante para el tema, siempre argumentando de qué manera éste aporta o soluciona el tema específico, se cita para conocer el proceso de análisis, sin llenar la disertación de citas de filósofos, pues éstas son solo una base para nuestra argumentación. También se recurre a otras afirmaciones que puedan ser más evidentes y que apoyen lo que se ha dicho. Analizar las consecuencias de diversas hipótesis y ver por qué son correctas, mostrar la coherencia de la afirmación que se ha hecho con otras afirmaciones que gozan de aceptación, mostrar los errores que se pueden seguir si se niega lo que se está afirmando y mostrar las razones que niegan la validez de las tesis contrarias. En todo el cuerpo de la disertación es necesario demostrar y tener una coherencia lógica pues no hay nada más catastrófico que llegar a una conclusión en donde hay contradicción, y no se debe avanzar a la siguiente etapa si no se ha establecido solidamente una argumentación, sin perder la disposición a refutarnos a nosotros mismos.

\section{- Conclusión:}

Se retoma la tesis inicial y se hace ver cómo todo lo que se ha dicho a lo largo de la disertación ha servido para confirmarlo, es como armar un rompecabezas en el que se ubica pieza por pieza para lograr al final vislumbrar la figura en su totalidad. Igualmente en la disertación debemos poco a poco ir armando la respuesta a la que queríamos llegar: "Antes de redactar la conclusión es necesario revisar el tema, el cual no debería perderse de vista nunca, con el fin de dar una respuesta coherente con la forma de la pregunta" ${ }^{3}$. Ahora, si realmente no se llega a responder la pregunta, debemos explicar a la contradicción que se llegó o decir por qué no se logró responderla, si el caso es que la respuesta está contenida dentro de otro problema más relevante, explicarlo y demostrar el porqué.

\section{ENSEÑANZA POR RESOLUCIÓN DE PROBLEMAS}

\section{Qué es una situación problémica:}

Se pueden denominar situaciones problémicas aquellos casos que generan preguntas que es necesario resolver, dado que estas situaciones generadoras de dudas llevan al individuo a desarrollar un pensamiento activo, creativo, productivo y autónomo necesario para fortalecer la búsqueda de la información, haciendo uso de nuevas estrategias y correlacionándolas con sus conocimientos previos, aunque debemos agregar que el nivel de dificultad de la situación problémica depende del conocimiento previo de cada estudiante.

3 GOMÉZ MENDOZA, Miguel Ángel. Didáctica de la disertación en la enseñanza de la filosofía: Métodos y procedimientos. Magisterio. Bogota, Colombia. 2005. p 40. 
Una situación problémica se puede denominar como un proceso productivo en que se formulan problemas prácticos basados en la realidad social, los cuales vislumbran cierta información permitiendo el desarrollo de preguntas, a su vez incitando a indagar sobre el caso planteado. Por lo tanto una situación problémica se origina cuando hay disponibilidad del individuo ante la necesidad de restaurar su pensamiento mediante procesos cognitivos, facilitando y contribuyendo al desarrollo de un problema, que, a su vez, originan nuevos problemas y por ende nuevas posibles soluciones: "Digamos entonces que la situación pedagógica que da lugar a preguntas que es necesario resolver, es la situación que se caracteriza por la existencia de un nuevo objeto de actividad intelectual y la aspiración a denominarlo por parte del sujeto del aprendizaje" ${ }^{4}$. Teniendo en cuenta que su base fundamental es el problema, ya que es el encargado de dinamizar al estudiante para que se comprometa como el responsable de desenredar aquellas situaciones problémicas en aras a brindar posibles soluciones a nuevos problemas que implican un nuevo reflexionar, estimulando a los estudiantes a la indagación poniendo a prueba ciertos niveles de comprensión necesarios en el desarrollo cognitivo del individuo.

\section{Características de la situación problémica:}

- Una de las principales características es que se le da prioridad al estudiante, ya que es el encargado de dilucidar la situación problémica de una forma activa, "jugando" de la mejor manera para conseguir los mejores resultados.

- El problema es basado en la realidad social para que integre a los jóvenes con el fin de que éstos reflexionen de una forma significativa, demostrando sus capacidades y a la vez reconociendo sus falencias.

- Crea incertidumbre, que combina la curiosidad con la duda, las cuales son las promotoras para que el joven se atreva a consultar hasta encontrar el meollo del asunto, convirtiéndose así en un pequeño investigador.

- La situación problémica es la base organizadora en la resolución de problemas, pues constituye una estructura generadora de preguntas que exigen su interpretación.

- Incita al estudiante a pensar ante la necesidad de comprender dicha situación pues sólo cuando hay preguntas hay un interés por buscar respuestas.

- La situación problémica, aunque es una situación real, es novedosa para el estudiante, ya que su aspecto es hipotético, incierto, atrayente a la investigación, dando así como resultado la construcción de nuevos conocimientos.

- La situación problémica no se presenta estructurada y organizada como una receta de cocina, sino que por el contrario presenta algo de complejidad y duda, en sí es algo confusa con cierto grado de dificultad intelectual.

- No podemos resolver ninguna situación problémica con la aplicación de una fórmula específica.

4 MEDINA GALLEGO, Carlos. La enseñanza problémica. Segunda edición. Editorial Rodríguez. Quito, Ecuador. P. 106 - 107. 
- El resultado de las situaciones problémicas siempre es la sumatoria de la gran diversidad de soluciones o respuestas encontradas a lo largo de la investigación.

- Las situaciones problémicas requieren de soluciones o respuestas argumentadas, motivando así la creatividad e innovación a la hora de jerarquizar, analizar y sintetizar la información consultada.

\section{Cómo implementarla}

Como ya mencionamos anteriormente la enseñanza por resolución de problemas es uno de los mejores métodos que se ajusta al entorno de un aprendizaje constructivista, pues responde a varios e importantes principios constructivistas tales como:

- Fomentar un aprendizaje que surja alrededor de problemas relevantes a los estudiantes

- Apoyar y guiar el trabajo de los estudiantes

- Valorar la expresión teniendo en cuenta el punto de vista de cada estudiante

- Promover la participación de grupos para que éstos puedan poner a prueba sus ideas contra otras posiciones o alternativas.

- Estimular el uso de fuentes de información.

\section{Diseño de la aplicación}

Se llevó a cabo teniendo en cuenta las siguientes fases:

1 FASE

* Problema: corresponde a la etapa en que el docente entrega a su grupo de estudiantes una guía que incluye la situación problémica que estos deben resolver.

* Clarificación de términos: Los estudiantes analizan la situación problémica y aclaran los términos desconocidos para ellos.

* Lluvia de ideas: En esta parte los estudiantes hacen un consenso con el fin de determinar qué tanto saben con respecto a la situación problémica.

* Formulación de objetivos de aprendizaje: Después de realizar el consenso los estudiantes están en la capacidad de aceptar qué ignoran y qué saben sobre el tema; con base en ello se planean las investigaciones necesarias.

2 FASE

* Trabajo de investigación: Los estudiantes se reúnen por grupos y hacen acuerdos sobre la forma como van a recolectar la información necesaria para dilucidar la situación problémica, ya sean entrevistas, internet o consulta en libros.

3 FASE

*Unificación de la información obtenida: Luego de obtenida la información los grupos se reúnen para unificar la información.

*Análisis y crítica: Los estudiantes exponen sus diferentes puntos de vista de acuerdo con el trabajo realizado y es acá donde se hace el análisis y selección que se integrará en el trabajo final.

*Trabajo final: es el escrito que presenta los argumentos más relevantes en la recolección de la información obtenida a lo largo del proceso. 
Implementación del Método de Disertación Filosófica en el Colegio de Boyacá

Este trabajo comienza realizando lecturas que toman como eje central el amor, esto con el propósito de empezar a dar herramientas a los estudiantes a la hora de escribir. Las lecturas básicas son: Lugar del amor en la vida humana de Beltrand Russell ${ }^{5}$, La amistad de Carlos García Gúal ${ }^{6}$, El arte de amar de Erick Fromm.

Dichas lecturas fueron evaluadas constantemente de forma escrita, para conocer el nivel de comprensión y los constantes avances en la misma. En la segunda fase se hizo primordial establecer las "reglas principales del juego", de tal manera que se explicó claramente qué es una disertación, cuál es el procedimiento a seguir, quá características lleva incluido este proceso y cuáles eran los objetivos a evaluar. Seguidamente los estudiantes procedieron a efectuar los primeros bosquejos sobre la disertación filosófica, resultado que se adquiere con el proceso que ellos adelantaron en cuanto a sus lecturas previas y demás investigaciones realizadas en diferentes fuentes tales como: diccionarios filosóficos, internet, textos filosóficos, entre otros.

Teniendo el escrito en el aula de clase se empezaron a organizar los primeros debates, que incluye los siguientes pasos:

- Lectura del texto escrito (disertación)

- Comentarios del grupo.

- Justificación de ideas por parte del lector.

- Conclusiones.

Este proceso se repite en cada uno de los estudiantes, quienes optan por afianzar su trabajo mediante las correcciones y recomendaciones hechas por el maestro a cargo del proceso, permitiendo así que ellos mejoren su nivel tanto argumentativo como escritural. Por último la docente valora el trabajo realizado por parte de los estudiantes teniendo en cuenta su participación y sus trabajos escritos con los requisitos ya mencionados anteriormente.

\section{Análisis y evaluación de la estrategia}

El paso a seguir consiste en señalar los principales puntos que se tuvieron en cuenta al desarrollo de la metodología la disertación filosófica:

- Rol asumido por el estudiante: En éste proceso el estudiante toma un papel mucho más activo y enérgico porque asume la responsabilidad de construir una disertación filosóficamente sostenible para ser argumentada y defendida frente a sus compañeros, con esto nos damos cuenta que bajo la estrategia disertación el joven participa critica e interpretativamente dentro de su contexto escolar, dándole las bases suficientes para ser un individuo activo socialmente.

- Rol asumido por el maestro: El maestro tiene la tarea de guiar y ayudar al estudiante para que fortalezca su proceso de aprendizaje, es así que el maestro asume el papel de orientador

5 RUSSELL, Beltrand. Antología. México: Siglo XXI, 1971. P. $91-98$.

6 GARCIA GUAL. Carlos. Epicuro. Madrid: Alianza Editorial, 1981. P. 210 - 218 
para reafirmar las capacidades adquiridas durante el proceso de formación. La filosofía nos puede servir de base para acercarnos a ellos.

- La evaluación: Dentro del proceso evaluativo el estudiante esta al día de sus adelantos cognitivos puesto que a medida que participa en clase y presenta sus primeros bosquejos de disertación se va dando cuenta de sus posibles falencias y fortalezas a la hora de escribir y de argumentar, de tal manera que tiene mas posibilidades de mejoramiento a lo largo del proceso.

\section{Implementación del Método de enseñanza por resolución de problemas en el Instituto Técnico Agropecualrio Rafael Uribe de Toca}

En la primera fase los estudiantes son organizados por grupos de ocho integrantes, en los que ellos mismos seleccionan un moderador y un secretario con el ánimo de que trabajen más ordenadamente para recibir por parte del maestro las situaciones problémicas las cuales llevan por título: "El turismo apasionado y mendigante del hincha", "Sabio no es el que escucha sino el que sabe escuchar, “¿Cuál será la decisión correcta?”

Llegando al otro punto que consiste en leer detalladamente la situación problémica para encontrar términos desconocidos, en caso de encontrarlos el docente deberá clarificar estos vocablos; a continuación los estudiantes proceden a contestar el cuestionario que está anexo al problema con el fin de conducirlos para que ellos mismos se interroguen qué tanto saben al respecto y qué deben averiguar en aras a dar posibles soluciones.

En la segunda fase los estudiantes por grupo realizan el trabajo de investigación y consulta, proceso que es desarrollado por medio de entrevistas a diferentes personalidades del entorno, profesores, libros, internet, en fin en cualquier fuente en donde obtuvieran información que contribuyera al proceso de solución de las situaciones problémicas.

En la fase final los estudiantes deben contar con la información suficiente para ahondar en el problema, es así que los estudiantes se ubican en círculo de tal forma que cada grupo pueda compartir sus experiencias e investigaciones realizadas para complementar el trabajo. Este debate se presenta en una forma secuencial y ordenada gracias a los moderadores y secretarios que son los encargados de tomar los apuntes que consideran relevantes para la ordenación y estructuración del trabajo final, en el cual se debe reflejar el proceso de aprendizaje.

La evaluación va paralela a las fases de la situación problémica, puesto que se tiene en cuenta el análisis y desarrollo del cuestionario anexo al problema, más la investigación realizada y el debate, que permite valorar los siguientes puntos: participación, apertura, riesgo, experiencia, sensibilidad. Se deja para el final del proceso la evaluación de la narrativa presentada por escrito, la cual debe reflejar lo que aprendió del tema.

\section{Análisis y evaluación de la metodología}

Exponemos a continuación los principales momentos o aspectos a la hora de la implementación del método: 
- Rol asumido por el estudiante: La enseñanza por resolución de problemas le da gran prioridad a los estudiantes puesto que les permite participar activamente de una manera autónoma, creativa e innovadora en el proceso de desarrollo de las clases, ya que son ellos los encargados de consultar y profundizar sobre los diversos temas. En una mayor parte la respuesta de los estudiantes fue propicia de acuerdo con las exigencias requeridas.

- Rol asumido por el maestro: Bajo la implementación de esta metodología el maestro debe asumir el papel de guía, pues es el encargado de orientar el proceso formativo de enseñanza que permite vislumbrar las capacidades cognoscitivas que tiene el estudiante y que el maestro debe ayudar a desarrollar y a dirigir para que lleven a la meta deseada.

- La evaluación: En esta parte se les dio a los estudiantes más independencia porque se valoró la habilidad que tenían para expresar sus propias ideas y argumentarlas frente a sus compañeros, de tal manera que se prescindió de la evaluación memorística, remplazándola por la construcción de un texto escrito por los mismos estudiantes; se hizo notorio el cambio evaluativo con respecto al que venía aplicándose en las clases de filosofía.

\section{Comparación de las dos metodologías}

$\mathrm{Al}$ analizar cuidadosamente las anteriores alternativas didácticas logramos apreciar que se presentan tanto similitudes como diferencias:

\section{Similitudes}

1. Las dos estrategias metodológicas fueron aplicadas de acuerdo con el contexto de cada institución puesto que cada colegio posee sus propios enfoques educativos.

2. Estas estrategias se constituyen bajo el enfoque constructivista, debido a que reconocen las capacidades cognitivas que cada estudiante tiene, por tal motivo lo que estas estrategias proponen es dar más participación y libertad al estudiante para que desarrolle sus competencias cognitivas dando como resultado un joven más autónomo, creativo, capaz de plasmar sus propias ideas por medio de argumentos coherentes y justificados, demostrando su actitud y habilidad investigativa, junto con su capacidad reflexiva.

2.1 Dichas metodologías tomaron en cuenta los preconceptos e ideas que los estudiantes tienen de cada tema, puesto que sus experiencias personales afectan de alguna u otra manera la aproximación y análisis al problema.

2.2 Al mostrar cierto grado de complejidad se hace necesaria la reflexión, conduciendo a los adolescentes a transitar por el camino de la duda, pues si hay duda siempre habrá algo que investigar y a su vez surgirá un nuevo conocimiento generador de nuevos interrogantes, también de posibles soluciones que desencadenan un pensamiento productivo capaz de aportar nuevas ideas solicitadas por una sociedad tan "moderna" y convulsionada como la nuestra.

3.3 En ambas metodologías nuestro papel de docentes se reflejó a lo largo del proceso educativo como un ente orientador. Aunque es necesario aclarar que no se adopta la postura del maestro que todo lo sabe, que pretende ahorrarle cualquier esfuerzo o angustia 
por pensar al estudiante, sino que se busca motivar al estudiante para que sea dueño de sus propias reflexiones, generando su progreso intelectual.

\section{Diferencias}

1. El abordaje al tema:

1.1 En la disertación filosófica antes de que los estudiantes abordaran el tema, el docente a cargo les fue aportando lecturas extraídas de obras filosóficas que contribuyeran a que los jóvenes fueran conociendo el tema a tratar, para que despertaran interés e investigaran por su parte más aportes antes de plantearles directamente la pregunta problémica.

1.2 En la enseñanza por resolución de problemas el maestro a cargo les hacia entrega de una lectura, en la que estaba implícito el tema que se pensaba tratar, presentado en forma de un problema real de la sociedad para que ellos lo analizaran y así pudieran responder el cuestionario anexo a ésta, lo cual les permitía interrogarse acerca de qué saben y qué no saben del tema a tratar conduciéndolos a una investigación más profunda sobre el tema.

2. La forma de trabajo:

2.1 En la disertación filosófica se privilegia el trabajo individual en cuanto a escritura e investigación se refiere, aunque se dedica una gran parte al debate en grupo, pero todas las disertaciones se presentaban y evaluaba de manera individual, esto para inducir a que cada estudiante argumentara y pensara por sí mismo sus propios planteamientos.

2.2 En la enseñanza por resolución de problemas la organización del trabajo fue grupal tanto en la investigación como en el trabajo escrito, mientras que en el debate se evaluaba la participación individual. El objetivo del trabajo en grupo era que cada estudiante aportara sus propias ideas y rescataran argumentos de las investigaciones para construir de esta forma el trabajo final.

A lo largo de esta exposición hemos vivido la realidad de cerca comprobando que el arte de enseñar no es tan fácil, pero aun así deja grandes satisfacciones construidas a través de las experiencias compartidas con los estudiantes, siendo ellos los gestores del proceso de cada una de nuestras experiencias como docentes: gracias a su participación y colaboración es que se pueden alcanzar grandes resultados 